\title{
Performance Evaluation of OFDM Coding System Using Concatenated BCH and LDPC Codes
}

\author{
H. Gagan1, R. Manas1, N. G. Girish Kumar² \\ ${ }^{1}$ Department of Electronics and Communication, Bangalore Institute of Technology, Bengaluru, India \\ ${ }^{2}$ Bangalore Institute of Technology, Bengaluru, India \\ Email: 1812gagan@gmail.com, manasreddy488@gmail.com,nggk_bit@live.in
}

How to cite this paper: Gagan, H., Manas, R. and Kumar, N.G.G. (2017) Performance Evaluation of OFDM Coding System Using Concatenated $\mathrm{BCH}$ and LDPC Codes. Int. J. Communications, Network and System Sciences, 10, 67-77.

https://doi.org/10.4236/ijcns.2017.105B007

Received: March 7, 2017

Accepted: May 23, 2017

Published: May 26, 2017

\begin{abstract}
This paper establishes a resilient concatenated coding platform for Orthogonal Frequency Division Multiplexing (OFDM) with Quadrature Amplitude Modulation (QAM) over Additive White Gaussian Noise (AWGN) channel. At the Forward Error Correction (FEC) coding unit, our proposed concatenated coding scheme utilizes standard type of $\mathrm{BCH}$ as external coding and LDPC as inner coding. In this scheme, Interleaver inclusion is seen as a catalyst to enhance the systems performance.
\end{abstract}

\section{Keywords}

Concatenated Codes, OFDM, LDPC, BCH, Interleaver

\section{Introduction}

Digital video broadcasting (DVB) [1] systems have stipulated the accomplishment of superior information rates, delivering efficient data transmission over error prone environment. Such systems utilize Orthogonal Frequency Division Multiplexing (OFDM) along with FEC (forward error correction) scheme that culminates in fast transmission incorporating M-ary QAM [2] modulation techniques. The high spectral efficiency of M-ary QAM and superior information rates is achieved by OFDM results in an efficient communication system.

By and large, the advantage of a multi-carrier system is that it can be largely unaffected by inter-symbol interference (ISI) by using pulse shaping filters. However, the OFDM system is extremely prone to frequency offset brought about by multipath delay spread channels. These frequency offsets impact the orthogonality of OFDM subcarriers, and it will likewise experience the inter-carrier interference (ICI). High data rates, along with spectral efficiency are 
achieved using OFDM which is an efficient technique to compensate rate loss caused by concatenation of codes [2]. OFDM strategies have served as basic building blocks for many of the current modulation schemes incorporated in Digital TV (DVB-T/T2, DVB-H, DMB-T/H, DVB-C2), Wireless LAN IEEE 802.11 a, IEEE 802.11 g, IEEE 802.11n, IEEE 802.11 ac, and IEEE 802.11 ad, Worldwide Interoperability for Microwave Access (WiMAX), Light Fidelity (Li-Fi), ADSL (Advanced Digital Subscriber Line), LTE Advanced 4G and 5G mobile phone standards, etc. [3].

The advent of Low Density Parity Check (LDPC) code was introduced by Gallager in 1963 [4]. Mostly ignored, Tanner's work in 1981 brought to light the robust LDPC technique, but during that time computers were not powerful enough and were forgotten for decades. In 1995 it was re-found by MacKay and Neal sparking a flurry of further research [5]. Gallager's simulation results demonstrated that these codes were processed on following the likelihood ratio or distribution of the extrinsic information exchanged during the message passing decoding standards, and it can approach Shannon limit while expanding code length. Besides, Gallager proposed a pragmatic iterative calculation that utilized extrinsic information traded between bit nodes and check nodes for decoding these codes. These codes can be decoded using various techniques ranging from high complexity to low complexity conversely providing satisfactory to exceptional error performance. Briefly, LDPC codes are linear block codes whose parity check matrix is exceptionally sparse. $\mathrm{BCH}$ code is a cyclic code that is built from Galois field. It was proposed by Hocquenghem in 1959, though done autonomously it was additionally found by Bose and Ray-Chaudhuri in 1960. In coding theory, concatenated codes form a class of error-correcting codes that are derived by combining an inner code and an outer code. They were conceived in 1966 by Dave Forney as a solution to the problem of finding a code that has both exponentially decreasing error probability with increasing block length and polynomial-time decoding complexity.

This paper implements Forward Error Correction (FEC) scheme using concatenation of $\mathrm{BCH}$ and LDPC codes, with $\mathrm{BCH}$ as the external encoder and LDPC as internal encoder for OFDM system with M-ARY QAM over AWGN channel [6] [7]. Also the variation in performance of the coding system with varied codeword lengths and check bit lengths is established.

This paper henceforth is organised in the following sections. Section 2 describes the proposed model as shown in Figure 1 along with the basic overview of the coding scheme and the algorithms incorporated. Section 3 illustrates simulation results juxtaposed with graphical representations to establish the intent of the topic and provide an analysis of the performance of the above incorporated coding system. Section 4 provides a conclusion to indicate the system's modern day relevance in terms of its performance parameters.

\section{System Description}

We have put forth an efficient scheme which involves the concatenation of 


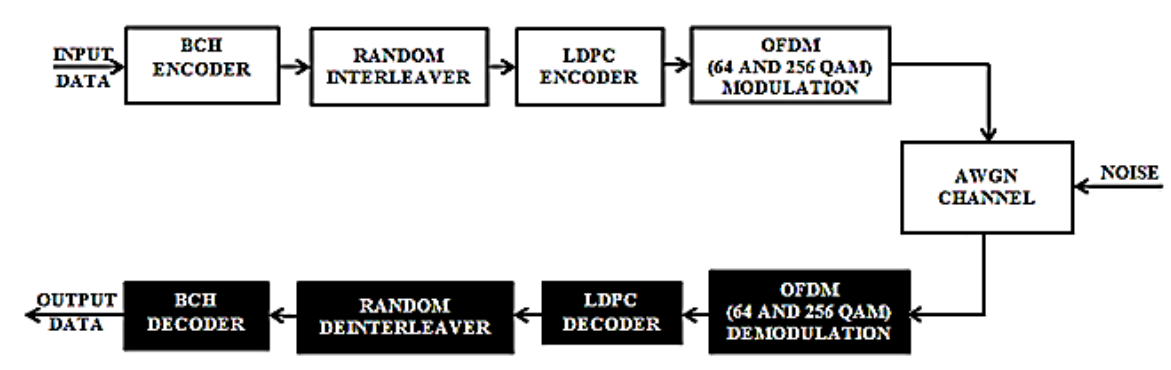

Figure 1. The concatenated coded OFDM based communication system.

LDPC codes with $\mathrm{BCH}$ codes, which is based on OFDM modulation over AWGN channel along with a random interleaving and de-interleaving scheme. In our proposed model we use a $\mathrm{BCH}$ encoder as the outer encoder and LDPC encoder as the inner encoder. The block diagram of the proposed system is as shown in Figure 1. We intend to compare the performance of the system with $\mathrm{M}$-ary QAM within OFDM. The Decoding algorithm for $\mathrm{BCH}$ used is Berlekamp-Massey algorithm and Sum product algorithm for the decoding of LDPC codes.

\section{A. $\mathrm{BCH}$ encoder}

These codes are a summed up type of Hamming codes that permits various error correcting capabilities. They shape a class of effective arbitrary error redressing cyclic codes which gives a determination of bigger block lengths, code rates and error revising capacity [6].

$\mathrm{BCH}$ codes are characterized by the following parameters:

For any positive integer value of $m$ where $m \geq 3$ and $t$ where $t$ is the error correcting capability variable indicated by $\mathrm{t}<2^{\mathrm{m}}-1$. A BCH code can be designed with the following parameters.

where,

$$
\text { Length of the code: } \mathrm{N}_{\mathrm{BCH}}=2^{\mathrm{m}}-1
$$

Total number of parity checks bits:

$\mathrm{N}_{\mathrm{BCH}}-\mathrm{K}_{\mathrm{BCH}} \leq \mathrm{mt}$

Minimum distance: $d_{\min } \geq 2 t+1$

This code is capable of correcting $t$ or fewer errors in a block of codes of size $\mathrm{n}$, this is called as t error correcting $\mathrm{BCH}$ codes. There is a need to determine the generator polynomial to define encoding technique. The generator polynomial is defined in terms of roots from the Galois field GF $\left(2^{\mathrm{m}}\right)$. The generator polynomial of the t-error rectifying $\mathrm{BCH}$ code of length $2^{\mathrm{m}}-1$ is the most reduced degree polynomial over GF(2) that has $\boldsymbol{\alpha}, \boldsymbol{\alpha}^{2}, \boldsymbol{\alpha}^{3}, \boldsymbol{\alpha}^{4}, \boldsymbol{\alpha}^{5}, \boldsymbol{\alpha}^{6}$ $\boldsymbol{a}^{2 t}$ as its roots. Let $\phi(x)$ be the minimal polynomial of $\boldsymbol{\alpha}^{\mathrm{i}}$. Then $\mathrm{g}(\mathrm{x})$ must be Least Common Multiple (LCM) of the product of $\phi_{1}(\mathrm{x}), \phi_{2}(\mathrm{x}), \ldots \ldots \phi_{2 \mathrm{t}}(\mathrm{x})$.

$$
\mathbf{g}(\mathbf{x})=\mathbf{L C M}\left(\phi_{1}(\mathbf{x}), \phi_{2}(\mathbf{x}), \phi_{3}(\mathbf{x}), \cdots, \phi_{2 \mathbf{t}}(\mathbf{x})\right)
$$

Since all the even powered values of $\alpha$ in the sequence $\alpha, \alpha^{2}, \alpha^{3}, \alpha^{4}, \alpha^{5}$, $\alpha^{6} \ldots \ldots \ldots \ldots, \alpha^{2 t}$ has the same minimal polynomial as the preceding odd power of $\boldsymbol{\alpha}$ in the sequence. Ignoring the minimal polynomials of the even power values 
of $\boldsymbol{\alpha}$ results in generator polynomial $\mathrm{g}(\mathrm{x})$ of the t-error correcting $\mathrm{BCH}$ code as

$$
\mathbf{g}(\mathbf{x})=\mathbf{L C M}\left(\phi_{1}(\mathbf{x}), \phi_{3}(\mathbf{x}), \phi_{5}(\mathbf{x}), \cdots, \phi_{2 \mathbf{t}-1}(\mathbf{x})\right)
$$

For example generator polynomial for a double error correcting code is:

$$
\mathbf{g}(\mathbf{x})=\mathbf{L C M}\left(\phi_{1}(\mathbf{x}), \phi_{3}(\mathbf{x})\right)
$$

Gives a $\mathrm{BCH}$ codes which is a $(15,7)$ cyclic codes

$$
\mathbf{d}_{\min } \geq 5
$$

Therefore the final Codeword is obtained by:

Codeword $=(\text { Message bits })^{\star}($ gen bits $)$

$$
\boldsymbol{C}=\boldsymbol{U} \boldsymbol{G}
$$

\section{B. $\mathrm{BCH}$ decoder}

The very well-known algorithm used for binary $\mathrm{BCH}$ codes is Berlekamp Iterative Decoding Algorithm and hence hereafter the algorithm implemented in the decoding technique [6].

By keeping the received polynomial $r(x)$ syndromes $S=\left(S_{1}, S_{2}, \ldots . . S_{2 t}\right)$ are calculated.

$$
\operatorname{rem}_{2 t}(x)=\frac{\varnothing_{2 t}(x)}{r(x)}
$$

where $t=$ error correcting Capability of code.

After finding the remainder polynomial syndromes are calculated by replacing $\mathrm{X}$ instead of $\alpha$

\section{Therefore}

$$
S=\left\{S_{1} S_{2}, S_{3}, \cdots, S_{2 t}\right\}
$$

After finding the syndromes, using the iterative procedure error Location polynomials $\sigma(x)$ are computed from these syndrome components $\mathbf{S}_{1}, \mathbf{S}_{2}, \ldots \ldots \mathbf{S}_{2 \mathrm{t}}$

$$
\sigma(x)=\sigma^{\mu+1}(x)=\sigma^{\mu}(x)+d_{\mu} d_{\rho}^{-1} x^{\mu-\rho} \sigma^{(\rho)}(x)
$$

where

$$
\begin{gathered}
d_{\mu+1}=S_{\mu+1}+\sigma_{1}^{\mu+1} S_{\mu+1}+\ldots+\sigma_{l_{\mu+1}^{\mu+1}} S_{\mu+2-l_{\mu+1}} \\
l_{\mu+1}=\max \left(l_{\mu}, l_{\rho}+\mu-\rho\right)
\end{gathered}
$$

where $\mu=-1,0, \cdots, 2 t, \rho=0,-1, \cdots, 2 t \quad$ (Table 1 ).

Then determine the error sets $\mathrm{e}(\mathrm{x})$.

The error sets are found by finding the roots of $\sigma^{2 t}(x)$.

Adding $\mathrm{e}(\mathrm{x})$ to the received polynomial $\mathrm{r}(\mathrm{x})$ we obtain the codeword

C. LDPC codes

LDPC codes are a class of linear block codes. In an $\mathrm{H}$ matrix of size $\mathrm{m}$ by $\mathrm{n}$ is low thickness in light of the way that the amount of $1 \mathrm{~s}$ in each row $\mathrm{w}_{\mathrm{r}}$ is $<<\mathrm{m}$ and the amount of $1 \mathrm{~s}$ in each column $\mathrm{w}_{\mathrm{c}}$ is $<<\mathrm{n}$. A LDPC is regular if $\mathrm{w}_{\mathrm{c}}$ is predictable for every column and $\mathrm{w}_{\mathrm{r}}=\mathrm{w}_{\mathrm{c}}(\mathrm{n}=\mathrm{m})$ is also reliable for each column. Else it is irregular [5]. In LDPC encoding, the codeword $\left(C_{0}, C_{1}, C_{2}, C_{3} \ldots C_{n}\right)$ involves the message bits $\left(M_{0}, M_{1}, M_{2} \ldots M_{K}\right)$ besides, some fairness check bits and 
Table 1. Initial decoding table to find error Location polynomial.

\begin{tabular}{ccccc}
\hline$\mu$ & $\sigma^{\mu}(x)$ & $d_{\mu}$ & $l_{\mu}$ & $\mu-l_{\mu}$ \\
\hline-1 & 1 & 1 & 0 & -1 \\
0 & 1 & $s 1$ & 0 & 0 \\
1 & & & \\
2 & & & \\
$\vdots$ & & & \\
$\vdots$ & & & \\
$2 t$ & & & \\
\hline
\end{tabular}

the scientific articulations are gotten from $\mathrm{H}$ network to create equity check bits. Their key great position is that they give an execution which is near as far as possible for a mixed bag of channels and straight time complex computations for disentangling. Other than they are suited for use that makes overpowering use of parallelism.

D. Matrix representation and Tanner Graph of LDPC codes

There are numerous ways codes can be represented in graph representation using coding theory. Tanner graph is the best way of representing LDPC codes in graphical format as shown in Figure 2. The tanner graph simplifies and gives good information about parity check matrix and also simplifies the explanation of decoding algorithm. As this graph consists of two opposite nodes tanner graphs can also be called as bipartite graphs.

For example:

Let us consider a parity check matrix $\mathrm{H}$ and the tanner graph for it as shown in the below diagram

$$
H=\left[\begin{array}{llllllll}
0 & 1 & 0 & 1 & 1 & 0 & 0 & 1 \\
1 & 1 & 1 & 0 & 0 & 1 & 0 & 0 \\
0 & 0 & 1 & 0 & 0 & 1 & 1 & 1 \\
1 & 0 & 0 & 1 & 1 & 0 & 1 & 0
\end{array}\right]
$$

\section{Parity check Matrix H}

\section{E. LDPC encoding}

Parity Check Matrix H: $\left(\mathrm{n}, \mathrm{w}_{\mathrm{c}}, \mathrm{w}_{\mathrm{r}}\right)$ : Parse Matrix

where

$\mathrm{n}$-number of rows

$\mathrm{w}_{\mathrm{c}}$-number of ones in column

$\mathrm{w}_{\mathrm{r}}-$ number of ones in row

Systematic Generator matrix $\mathrm{G}$ is obtained by doing row operations on $\mathrm{H}$ matrix.

where

$$
\mathbf{G}=\left[P_{(\mathbf{n}-\mathbf{k}) * \mathbf{k}} I_{\mathbf{k} * \mathbf{k}}\right]
$$

where $\mathrm{K}$ is the size of message bits. ( $\mathrm{P}$ is a dense matrix) and 


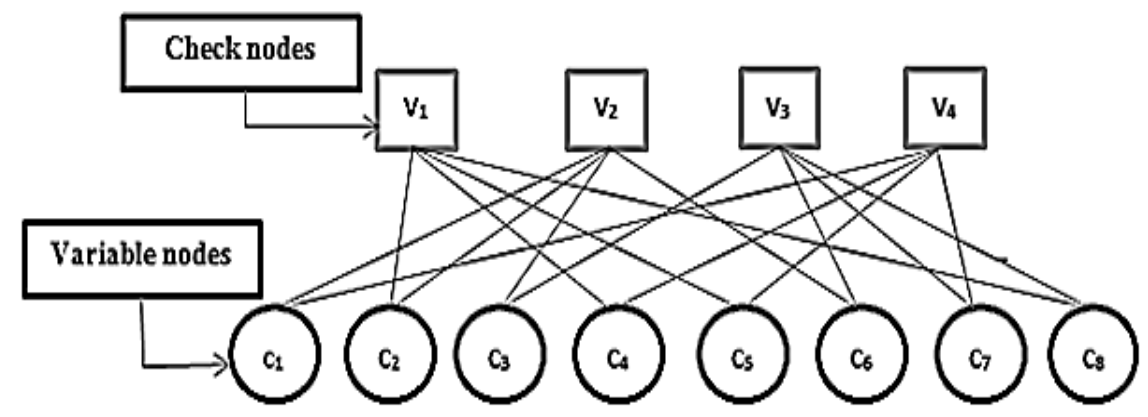

Figure 2. Tanner graph of $\mathrm{H}$.

$$
\mathbf{G} H^{T}=0
$$

If we have message $\mathrm{m}$ of $\mathrm{k}$ bits then the codeword is obtained by

$$
\mathbf{C}=\left[\mathbf{m ~ m} P^{T}\right]
$$

$>$ Complexity of encoding is a function of $n^{3}$ where $n$ is the number of XOR operations done between rows to generate a generator matrix

F. LDPC decoding

There are several novel methods used in decoding LDPC codes. The most common one are Bit Flipping Algorithm (BFA) and Sum Product Algorithm (SPA). The following illustration gives knowledge on Bit Flipping Algorithm which is normally based on hard decision decoding and Sum Product algorithm based on soft decision decoding.

\section{Sum product algorithm}

Soft decision decoding of LDPC codes, which depends on the idea of belief propagation yields in a superior decoding performance and therefore belief propagation is the preferred decoding algorithm for LDPC codes. The soft decision decoder works with the same rule as the hard-choice decoder, aside from that the messages are the conditional probability that the received bit is a 1 or a 0 based on the given received vectory. Let $\mathrm{Pi}=\mathrm{P}_{\mathrm{r}}\left(\mathrm{c}_{\mathrm{i}}=1 \mid \mathrm{y}_{\mathrm{i}}\right)$ be the conditional probability that $c_{i}$ is a 1 given the estimation of $y$ and let $P_{r}\left(c_{i}=1 \mid y_{i}\right)=1-P_{i} \cdot q_{i j}$ is a message sent by the variable node $c_{i}$ to the check node $v_{j}$. Each message contains dependably the pair $\mathrm{q}_{\mathrm{ij}}(0)$ and $\mathrm{q}_{\mathrm{ij}}(1)$ which remains for the amount of belief that $y_{i}$ is a " 0 " or a " 1 ".

$\mathrm{r}_{\mathrm{ji}}$ is a message sent by check node to variable node. There is rji(0) and rji(1) that indicates the amount of belief in that yi is a " 0 " or a " 1 " [8].

Step 1: Compute the initial value of Log Likelihood Ratio (LLR) transmitted from the variable node $C_{i}$ to check node $V_{i}$ for all I where $1 \leq i \leq n$

$$
L\left(q_{i j}\right)=L\left(V_{i}\right)=\frac{2 y_{i}}{\sigma^{2}}=L L R_{i}=\log \frac{P\left(V_{i}=0 \mid y_{i}\right)}{P\left(V_{i}=1 \mid y_{i}\right)}
$$

where $\sigma^{2}$ denotes the channel noise variance and $P\left(C_{i}=0 \mid y_{i}\right)$ denotes the probability value for a given input $y_{i}$

Step 2: Find $L\left(r_{i j}\right)$ transmitted from the check node $C_{i}$ to variable node $V_{i}$ for all $i ; 1 \leq i \leq n$ 


$$
L\left(r_{i j}\right)=\prod_{i^{\prime} \in \frac{V_{j}}{i}} \alpha_{i^{\prime} j} \phi\left(\sum_{i^{\prime} \in \frac{V_{j}}{i}} \phi\left(\beta_{i^{\prime} j}\right)\right)
$$

where

$$
\begin{gathered}
\alpha_{i^{\prime} j}=\operatorname{sgn}\left(L\left(q_{i j}\right)\right) \\
\beta_{i j}=\left|L\left(q_{i j}\right)\right| \\
\phi(x)=\log \left(\frac{e^{x}+1}{e^{x}-1}\right)
\end{gathered}
$$

Step 3: After computing $L\left(q_{i j}\right)$ it is necessary to find the modification of $L\left(q_{i j}\right)$ which is used to find the data transmitted from the variable node $V_{i}$ to check node $S_{i}$ for all i where $1 \leq i \leq n$.

$$
L\left(q_{i j}\right)=L\left(S_{i}\right)+\sum_{j^{\prime} \in \frac{S_{i}}{j}} L\left(r_{j^{\prime} i}\right)
$$

Step 4: The soft output obtained can be represented as:

$$
L\left(Q_{i}\right)=L\left(V_{i}\right)+\sum_{j^{\prime} \in S_{i}} L\left(r_{j i}\right)
$$

Step 5: Once we obtain the soft output then hard decision is made to figure out the output by

$$
\hat{C} w_{i}=\left\{\begin{array}{l}
1 L\left(Q_{i}\right)<0 \\
0 \text { otherwise }
\end{array}\right.
$$

It is found that the soft decision decoding method for LDPC decoder gives much better result compared to hard decision decoding. Therefore soft decision decoding method is chosen for decoding using LDPC in the proposed research work.

\section{Simulation Results}

The proposed scheme's performance was investigated using MATLAB which involves the transmission of an image through a Forward Error Correction coding system whose integrity depends on OFDM with M-ary QAM transmitted through an AWGN channel. The scheme proposed here implements 4K OFDM with 128 sub-frames and the simulated results for an un-coded system are given in Figure 3, where we observe Bit error rates ranging from $10^{-0.48}$ to $10^{-0.68}$ for decreasing order of M-ary QAM. The simulated results as shown in the Figure 4 represent the received image, where adistorted image is obtained even after reaching higher SNR values. Figure 5 is a graph of BER vs SNR for coded system without an Interleaver for various values of $\mathrm{M}(4,8,16,32,64,128,256)$. Here BER values drop to almost $10^{-3}$, hence significant error correcting capabilities which result in an undistorted image as shown in Figure 6. The system establishes high coding gain as seen from the graphs shown in Figure $\mathbf{3}$ and Figure $\mathbf{5}$. We also noticed that by implementation of $\mathrm{BCH}$ as the outer decoder reduced error floor at comparatively higher SNRs. In this coding system the LDPC decoder in the concatenated scheme has increased the error correcting capability. 


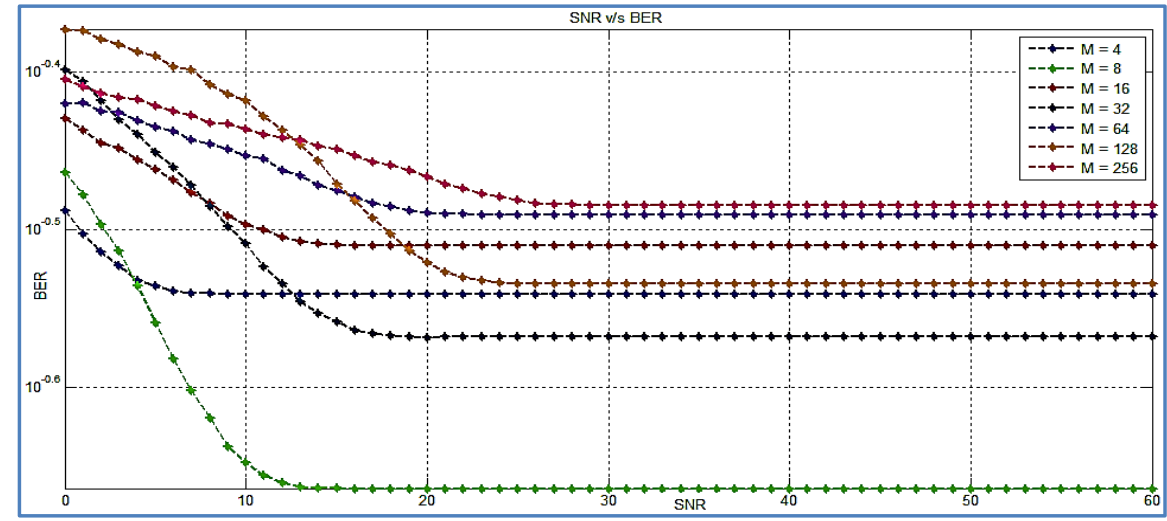

Figure 3. Uncoded scheme for M-ary QAM using OFDM.

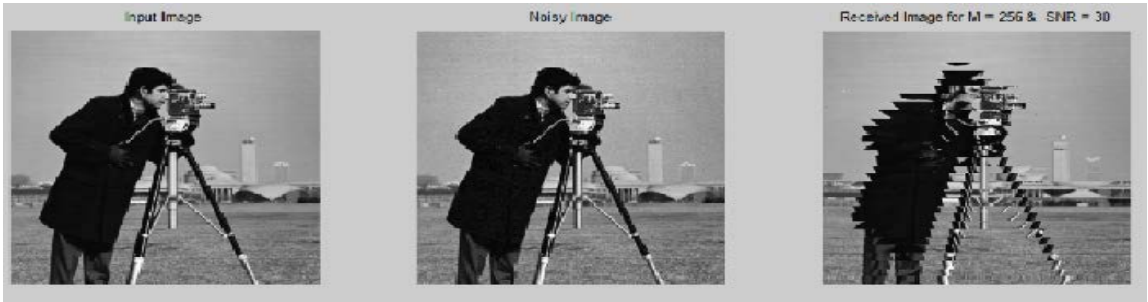

Figure 4. Image received for an uncoded scheme.

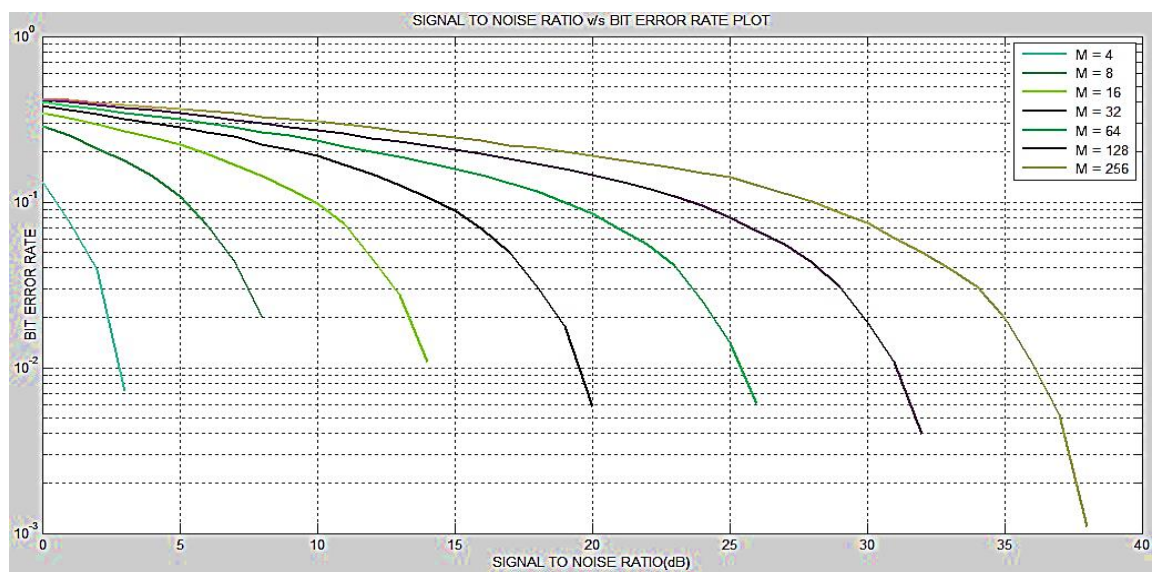

Figure 5. Concatenated coding scheme for M-ary QAM using OFDM without Interleaver.

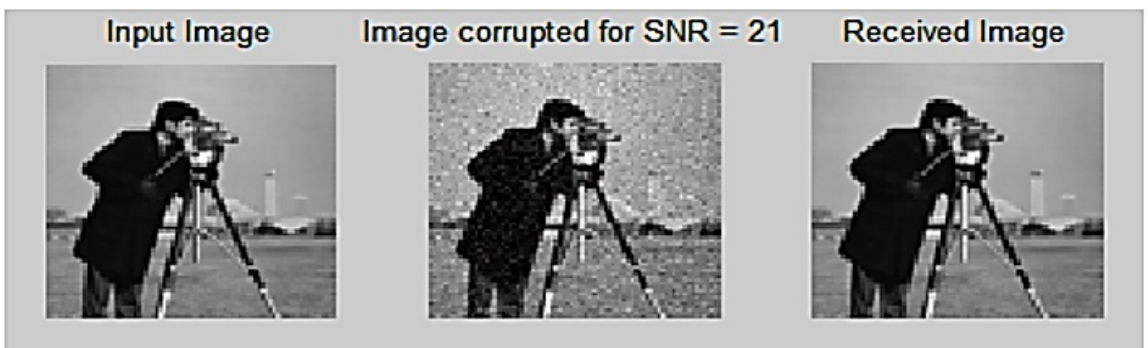

Figure 6. Image received for a coded scheme.

Inclusion of the Interleaver in this proposed scheme reduces burst errors and thus allows higher error correcting capability of the concatenated coding system. 
Concatenated codes inevitably lead to rate loss and thus decreasing its spectral efficiency. The OFDM system implemented in our proposed scheme compensates for this rate loss because of its high spectral efficiency. The BER increases gradually as SNR values increase and this scheme is suitable for higher order M-ary QAM (64QAM and 256 QAM).The considered LDPC codes parity check matrix corresponds to an irregular LDPC code with a code rate $=1 / 2(32,400 /$ 647800) where the block length of the code is 64,800 .

Our further research includes performance evaluation of 256-QAM using an Interleaver as shown in Figure 7. We have found that the BER has significantly been enhanced resulting in $10^{-6}$. On comparing a coded system with Interleaver and one without an Interleaver, a difference of around $15 \mathrm{~dB}$ was seen at a BER of $10^{-3}$

Figure 8 shows simulation results using different values of code word length denoted by $\mathrm{n}=255,63,15$ and message length denoted by $\mathrm{k}=247,57,5$ with error correcting capability denoted by $\mathrm{t}=1,1,3$ respectively. The following per-

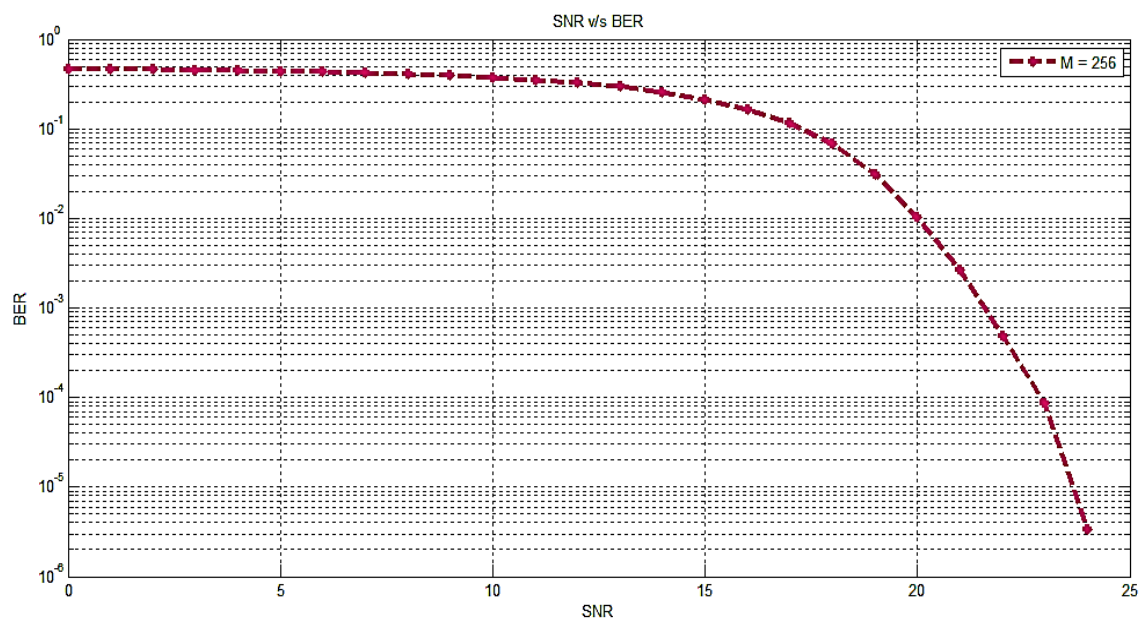

Figure 7. Concatenated coding scheme for 256-QAM using OFDM with Interleaver.

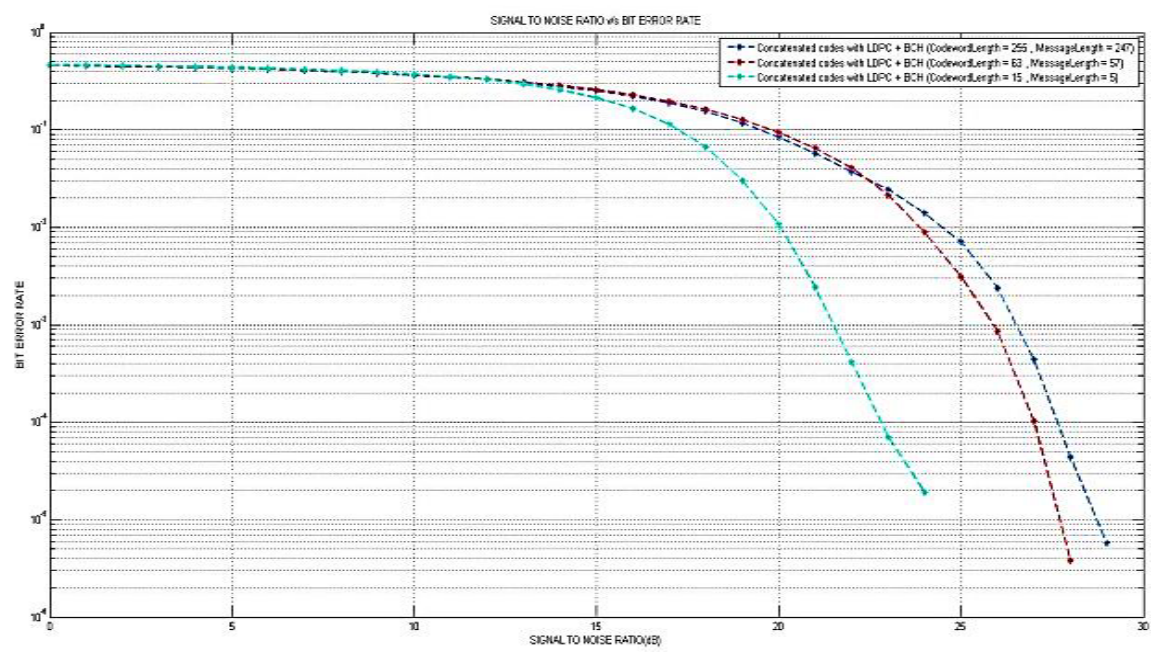

Figure 8. Concatenated coding scheme for different $\mathrm{BCH}$ code rates using OFDM and 256 QAM. 
formance of different $\mathrm{n}, \mathrm{k}$ values as shown in the Figure 8 indicate that larger codeword length exhibit higher SNR values. Both the single error correcting capability codes exhibit a bit error of around $10^{-6}$ at $28 \mathrm{~dB}$ SNR. However the triple error correcting code exhibits an bit error rate of near $10^{-5}$ at low SNR of $24 \mathrm{~dB}$. A balance must be struck between codeword length $n$, error correcting capability $\mathrm{t}$ and $\mathrm{SNR}$ to meet various requirements.

\section{Conclusions}

This paper establishes that a system involving the concatenation of LDPC and $\mathrm{BCH}$ codes provides good error correcting capabilities at moderately low SNR values. In this paper the new coding scheme is investigated and it is seen that the concatenation of codes diminishes the effect of error floor, simultaneously enhancing the coding system's error correcting capability. The concatenation of codes inevitably leads to rate loss and decrease spectral efficiency, OFDM's inclusion compensates for the prior problem thus making it one of the most effective systems for present day and future solutions in digital video broadcasting applications.

Further we are planning to enhance the performance of the above concatenated coding scheme by using polar codes replacing LDPC codes. Polar codes are extended version of non-binary $\mathrm{BCH}$ codes which uses successive cancellation algorithm for successful decoding. These codes can outperform LDPC and Turbo codes.

\section{Acknowledgements}

The authors thank DR A.G. Nataraj, Principal, Bangalore Institute of Technology, Bengaluru, India for his support and encouragement and Dr. Sree Ranga Raju M N, Professor, Department of Electronics and Communication Engineering, Bangalore Institute of Technology, Bengaluru, India for his constant support and guidance throughout the course of preparing this paper.

\section{References}

[1] Zheng, J.-Y. and Gao, J. (2012) Turbo Coding in DVB-RCS Standard over the Stratospheric Communication Channel. IEEE World Congress on Information and Communication Technologies, 1047-1051.

[2] van Wyk, J. and Linde, L. (2007) Bit Error Probability for a M-ary QAOFDM-Based System. IEEE AFRICON2007, September 2007, 1-5.

[3] Iqbal, Z. and Nooshabadi, S. (2012) Effects of channel Coding and Interleaving in MIMO-OFDM Systems. IEEE 54th International Midwest Symposium on Circuits and Systems (MWSCAS), August 2012, 1-4.

[4] Gallager, R.G. (1963) Low-Density Parity-Check Codes. IEEE Trans. Inform. Theory, 8, 21-28. https://doi.org/10.1109/TIT.1962.1057683

[5] MacKay, D.J.C. and Neal, R.M. (1996) Near Shannon Limit Performance of Low Density Parity Check Codes. Electron. Lett., 32, 1645-1646. https://doi.org/10.1049/el:19961141

[6] Lin, S. and Costello, D.J. (2004) Error Control Coding. 2nd Edition, Prentice-Hall, 
Englewood Cliffs.

[7] Moon, T.K. (2005) Error Correction Coding Mathematical Methods and Algorithms. Wiley Publication. https://doi.org/10.1002/0471739219

[8] Davey, M.C. and MacKay, D.J.C. (1998) Low Density Parity Check Codes over GF(q). Proc. Inf. Theory Workshop, 70-71. https://doi.org/10.1109/itw.1998.706440

Submit or recommend next manuscript to SCIRP and we will provide best service for you:

Accepting pre-submission inquiries through Email, Facebook, LinkedIn, Twitter, etc. A wide selection of journals (inclusive of 9 subjects, more than 200 journals)

Providing 24-hour high-quality service

User-friendly online submission system

Fair and swift peer-review system

Efficient typesetting and proofreading procedure

Display of the result of downloads and visits, as well as the number of cited articles Maximum dissemination of your research work

Submit your manuscript at: http://papersubmission.scirp.org/

Or contact ijcns@scirp.org 